\title{
Pressure-Enabled Drug Delivery Approach in the Pancreas with Retrograde Venous Infusion of Lipiodol with Ex Vivo Analysis
}

\author{
Aravind Arepally ${ }^{1}$ (D) James Chomas ${ }^{2} \cdot$ Steven C. Katz $^{3,4} \cdot$ David Jaroch $^{5}$ • \\ K. Pallav Kolli ${ }^{6} \cdot$ Ethan Prince $^{7} \cdot$ Robert P. Liddell $^{8}$
}

Received: 20 April 2020/Accepted: 7 August 2020/Published online: 7 September 2020

(C) The Author(s) 2020

\begin{abstract}
Purpose To determine the safety and feasibility of pancreatic retrograde venous infusion (PRVI) utilizing a microvalvular infusion system (MVI) to deliver ethiodized oil (lipiodol) by means of the Pressure-Enabled Drug Delivery (PEDD) approach.

Methods Utilizing transhepatic access, mapping of the pancreatic body and head venous anatomy was performed in 10 swine. PEDD was performed by cannulation of veins in the head $(n=4)$ and body $(n=10)$ of the pancreas with a MVI (Surefire ${ }^{\circledR}$ Infusion System (SIS), Surefire Medical, Inc (DBA TriSalus ${ }^{\mathrm{TM}}$ Life Sciences)) followed by infusion with lipiodol. Sets of animals were killed either immediately $(n=8)$ or at 4 days post-PRVI $(n=2)$. All pancreata were harvested and studied with micro-CT and histology.
\end{abstract}

Aravind Arepally

arepally@raadocs.com

1 Division of Interventional Radiology, Piedmont Radiology, Piedmont Healthcare, 1984 Peachtree Road, Suite 505, Atlanta, Georgia 30309, USA

2 Formerly TriSalus Life Sciences, Inc, Westminster, USA

3 Office of Therapeutic Development and Department of Surgery, Roger Williams Medical Center, Providence, USA

4 Department of Surgery, Boston University School of Medicine, Boston, USA

5 TriSalus Life Sciences, Inc, Westminster, USA

6 Department of Radiology and Biomedical Imaging, University of California, San Francisco, USA

7 Radiology, Roger Williams Medical Center, Providence, USA

8 Russell H. Morgan Department of Radiology and Radiological Science, Johns Hopkins University, Baltimore, USA
We also performed three-dimensional volumetric/multiplanar imaging to assess the vascular distribution of lipiodol within the glands.

Results A total of 14 pancreatic veins were successfully infused with an average of $1.7(0.5-2.0) \mathrm{mL}$ of lipiodol. No notable change in serum chemistries was seen at 4 days. The signal-to-noise ratio (SNR) of lipiodol deposition was statistically increased both within the organ in target relative to non-target pancreatic tissue and compared to extra pancreatic tissue $(\mathrm{p}<0.05)$. Histological evaluation demonstrated no evidence of pancreatic edema or ischemia.

Conclusions PEDD using the RVI approach for targeted pancreatic infusions is technically feasible and did not result in organ damage in this pilot animal study.

Keywords Locoregional therapy · Pancreas .

Pancreatic vein $\cdot$ Lipiodol

$\begin{array}{ll}\text { Abbreviations } \\ \text { ALT } & \text { Alanine aminotransferase } \\ \text { MVI } & \text { Microvalve infusion catheter } \\ \text { AST } & \text { Aspartate aminotransferase } \\ \text { IRE } & \text { Irreversible electroporation } \\ \text { PRVI } & \text { Pancreatic retrograde venous infusion }\end{array}$

\section{Introduction}

Pancreatic ductal adenocarcinoma (PDAC) has one of the highest cancer case fatality rates with an average 5-year overall survival (OS) of $\leq 8 \%$ for patients with advanced disease [1]. Its insidious development and aggressive biological behavior, with resistance to multimodal therapy, have made improvements in patient outcomes challenging 
to achieve. Combination [2] and neoadjuvant [3, 4] chemotherapy modestly improves median OS by about 8 16 weeks in advanced disease [5]. Localized delivery via intra-arterial (IA) chemotherapy, with the goal of achieving high local drug concentrations while maintaining low systemic drug level, has been reported but may be impractical in the majority of patients due to anatomical challenges $[6,7]$.

The highly desmoplastic architecture of PDAC leads to the relative inability of chemotherapy to penetrate the tumor even when delivered regionally via IA infusion [8] due to the presence of a dense physical barrier [9]. This same stromal architecture also results in tumors being supplied by very small feeding arteries with minimal perfusion capacity [10]. Furthermore, high solid stress and interstitial fluid pressure in excess of $130 \mathrm{mmHg}$ result in the physical compression of vasculature within the PDAC tumor mass and suppression of normal convective flux of molecules from the blood stream to the tissue $[11,12]$. Thus, the physical and anatomical features associated with PDAC limit the effectiveness of local IA delivery by conventional means.

To circumvent the challenges and limitations of local IA and systemic chemotherapy in PDAC, we postulate that pancreatic retrograde venous infusion (PRVI) may enable improved locoregional PDAC drug delivery, while mitigating off-target toxicity. As there are smaller arterial feeders which are often not accessible, the venous approach may overcome this limitation in pancreatic adenocarcinoma $[10,13]$. We hypothesize that pressurization of the venous drainage by means of a microvalvular catheter will allow for retrograde infusion directly into the tumor vasculature. The technique, termed Pressure-Enabled Drug Delivery (PEDD), is expected to result in improved selectively, isolation, and perfusion of target tissue within the pancreas.

To test the PEDD approach, we systematically evaluated ethiodized oil (lipiodol; Guerbet, Paris, France) distribution in normal swine pancreata. The primary objective of this study was to assess the safety and feasibility of PEDD with retrograde venous infusion via microvalvular infusion catheters (MVI) and to quantify the distribution of infusate.

\section{Materials and Methods}

\section{Animal Model}

All animal experiments were performed under the supervision and with approval of the T3 Research Institute Animal Care and Use Committee (AAALAC accredited). We tested healthy, growing swine $(50-60 \mathrm{~kg}, n=10)$. All were sedated with an intramuscular injection of a mixture of ketamine $(22 \mathrm{mg} / \mathrm{kg})$, acepromazine $(1.1 \mathrm{mg} / \mathrm{kg})$, and atropine $(0.05 \mathrm{mg} / \mathrm{kg})$. A single dose of antibiotics, DualCillin $(300,000 \mathrm{U} / \mathrm{mL}$, i.m.), was administered prior to the interventional procedures. Intravenous pentobarbital ( $20 \mathrm{mg} / \mathrm{kg}$ body wt) was used to induce the animal after which the animals were intubated and mechanically ventilated with $2 \%$ isoflurane and $98 \%$ oxygen.

\section{Pressure-Enabled Drug Delivery (PEDD) with Microvalvular Infusion System}

The Surefire ${ }^{\circledR}$ Infusion System (SIS) (Surefire Medical, Inc (DBA TriSalus ${ }^{\mathrm{TM}}$ Life Sciences), Westminster, CO) is a $3.4 \mathrm{~F}$ coaxial infusion microcatheter having a $0.025^{\prime \prime}$ inner lumen with a microvalve at the distal end that serves as the conduit for physician-specified agents. The distal funnelshaped microvalve is manufactured in two sizes, covering vessels ranging from $2-4.0 \mathrm{~mm}$ ( $025 \mathrm{M}$ configuration) and 4.0-6.0 mm (025L configuration).

\section{Pancreatic Venous Anatomy Evaluation (Figs. 1 and 2)}

The study was performed in ten swine. Angiography was performed on a Phillips system, Integra Flat Panel. Percutaneous access to the portal vein was achieved using ultrasound guidance with a transhepatic technique. A 22-gauge Chiba needle (Neff Percutaneous Access Set; Cook, Bloomington, Ind) was placed into a branch of the right portal venous system. After the portal vein was accessed, a 5-French sheath (Cordis, Miami, FL) was placed in the main portal vein. Flush portography was performed with a 5-F angiographic pigtail catheter placed in the splenic vein. Using standard 5 French angiographic catheters (Omni Sos, Angiodynamics, Queensbury, NY), mapping of the pancreatic venous anatomy was performed to identify the venous drainage of the pancreatic body/head and to assess for collateral venous drainage. Venous collaterals, when present, were embolized using coils (6-8 mm Nester Embolization Coils; Cook, Bloomington, Ind) and gelfoam to limit non-targeted delivery of lipiodol. Next, cannulation of the main pancreatic venous drainage of the head $(n=4)$ and body $(n=10)$ was performed with the MVI system. Subselective venograms were performed after devices were deployed in the head or body to confirm appropriate positioning. Next, second-order branches were selectively catheterized utilizing diagnostic catheters and the MVI systems with 0.016" Fathom guidewires (Boston Scientific, Marlborough, MA). Pancreatic vein diameters were measured in the main and second-order branches, and flow dynamics were assessed in the distal vascular bed after the MVI systems were deployed.

A total of 14 PRVIs were performed in a total of 10 swine (pancreatic head $=4$ and body $=10$ ). The endpoint 
of embolization was of stasis or sluggish flow with leakage of lipiodol through the MVI system. After infusion of ethiodized oil (lipiodol; Guerbet, Paris, France), catheters were flushed with a $5 \mathrm{cc}$ of saline to clear any residue at the tip. After the procedure, all catheters and sheaths were removed. In the survival animals $(n=2)$, the access to the portal vein was closed by a transhepatic deployment of a Mynx Vascular Closure Device (AccessClosure, Inc, Santa Clara, CA). Two animals were killed 4 days post-RPVI. Eight animals were killed immediately post-RPVI. All animals were humanely euthanized by an IV injection of barbiturates (e.g., $100 \mathrm{mg} / \mathrm{kg}$ pentobarbital) while under general anesthesia followed by potassium chloride to arrest the heart in end diastole. All pancreata were then harvested for analysis.

\section{Biochemical Analysis}

Two swine underwent standard laboratory assessment preprocedure, post-procedure, $24 \mathrm{hr}$, and $96 \mathrm{hr}$ (4 days) postinfusion prior to explant. This included standard comprehensive metabolic panel, complete blood count, and amylase analysis.

\section{Ex Vivo Lipiodol Distribution Analysis}

After the infusion procedure, high-resolution images of the explanted pancreata were analyzed for lipiodol distribution pattern, clumping, and extension of lipiodol into non-target regions in the pancreas, duodenum, and gallbladder. All explanted pancreata and duodenums underwent micro-CT imaging (GE eXplore CT $120^{\mathrm{TM}}$ System, Milwaukee, WI). 3D volumetric/multiplanar MIP images of were performed utilizing an Osirix Workstation (Osirix 3.8.1, 64 bit, Geneva, Switzerland). Data sets were generated for each pancreas $(n=10)$ and explanted duodenum and gallbladder (whole coronal plane, whole sagittal plane, whole axial plane) for 3D volume-rendered MIP and multiplanar reconstructions (MPR) images with 1-mm slice thickness (approximately 25 slices per pancreas). To obtain attenuation values of the tissue, each slice of the micro-CT image was aligned to the midline of each pancreas, and a region of interest was placed in the area of maximum density. Ten regions of interest were generated in the body and head of the pancreas in the areas of infusion. To achieve consistency, a circular region of interest (ROI) was used, and the size was adapted to analyze a $4-\mathrm{mm}^{2}$ portion of the pancreas. In a similar manner, circular ROIs were used in extra pancreatic tissue (explanted gallbladder and duodenum). Quantitative assessment of the signal-to-noise ratio (SNR) in various parts of the pancreas (head, body/tail) and extra pancreatic tissue was measured using circular ROIs. The ROI and standard deviation (SD) for the background were also obtained. The SNR values for the pancreatic head, tail, and extra pancreatic tissue (duodenum and gallbladder images) were calculated using the mean pixel intensity (I) of the ROI in the target tissue divided by the SD of background, the "estimated noise."

$\mathrm{SNR}(\mathrm{ROI})=\operatorname{Mean} \mathrm{I}(\mathrm{ROI}) \div \mathrm{SD} \mathrm{I}$ (noise)

Statistical analysis was performed using an unpaired t-test to compare the SNR of the pancreatic head (target) to tail (non-target) and the SNR of pancreatic tissue to extra pancreatic tissue. Analysis was conducted using GraphPad Prism (GraphPad Software, San Diego, California).

\section{Histopathological Analysis}

Several one-gram tissue samples were collected from the infusion region within the pancreas and from the other regions of interest in the gallbladder and duodenum adjacent to the infusion site. The tissues were then fixed in $10 \%$ neutral-buffered formalin. The fixed samples were then sent to HistoWiz (Brooklyn, NY) for staining and analysis. Histology was performed by HistoWiz according to Standard Operating Procedures. Submitted tissues were sectioned $\sim 10$ um thick and stained for hematoxylin and eosin (H\&E) histology stain. Whole slide scanning (40X) was performed on an Aperio AT2 (Leica Biosystems).

Microscopic evaluation and morphometry were performed by trained personnel. Scanned slide images were utilized for microscopic evaluation and photomicrographs.

\section{Results}

\section{Pancreatic Venous Anatomy Evaluation (Figs. 1, 2 and 3)}

A total of 14 PEDD procedures were conducted via PRVI in a total of ten swine without any complications. Due to the presences of collaterals arising off the inferior pancreaticoduodenal branches, embolization of these vessels was necessary to target pancreatic head infusions. With pancreatic body infusions, the main pancreatic vein draining into the splenic vein was utilized, with no significant collaterals detected.

In 4 swine, angiography revealed vasospasm or small vascular perforations in the side branch vessels related to guidewire manipulation which did limit therapeutic delivery. An average of $1.7 \mathrm{~mL}$ (range $0.5-2.0 \mathrm{~mL}$ ) of lipiodol was infused in each target. Micro-CT provided high-resolution visualization of the pancreatic parenchyma with $70 \mu \mathrm{m}$ isotropic resolution. Lipiodol deposition was present in the deep pancreatic parenchyma as confirmed by micro-CT but not always readily visible under fluoroscopy. 


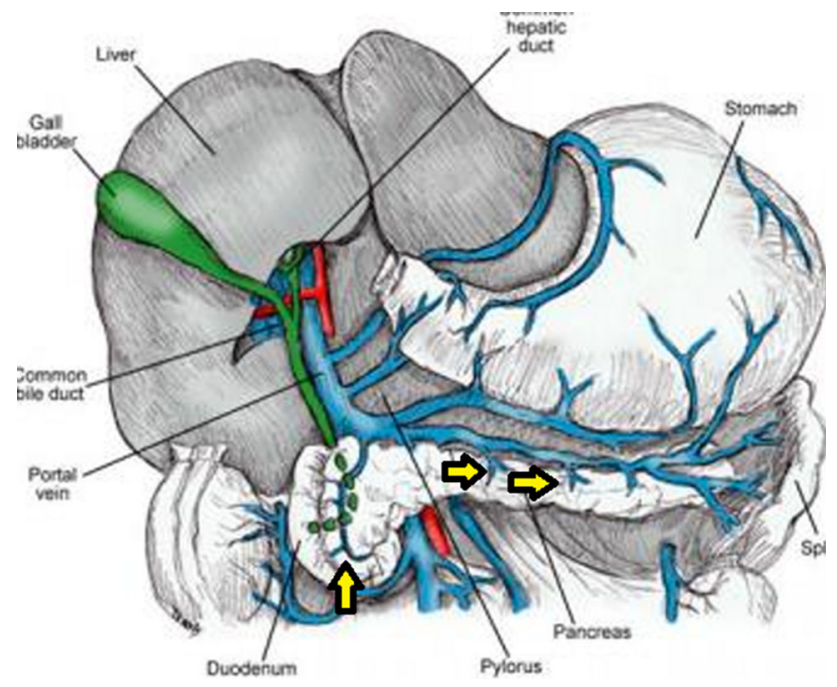

Fig. 1 Artistic rendering of pancreatic venous anatomy (Image reproduced with permission from Medscape Drugs \& Diseases (https://emedicine.medscape.com/), Pancreas Anatomy, 2017, available at: .) Yellow arrow $=$ pancreatic veins

\section{Ex Vivo Qualitative Analysis}

3D MIP and 3D MPR images generated from micro-CT data sets showed the distribution of lipiodol predominately in the target tissue bed of the pancreas body or tail (Figs. 2C and 3D). In contrast, PRVI into the pancreatic body demonstrated accumulation of lipiodol in the pancreatic tail and head suggestive of intra-pancreatic collaterals which were not readily visible at the time of procedure. PRVI delivery of lipiodol to the pancreatic head resulted in a low degree of non-target accumulation present in the duodenum and gallbladder (Fig. 3D). Qualitative examination of the target pancreatic vascular bed showed a distribution of the lipiodol throughout the parenchyma with extension into the deeper tissue parenchyma.

\section{Biochemical Analysis (Table 1)}

Laboratory values from 2 animals did not display notable changes from pre-procedure baselines at the postprocedure, 24-hr, and 96-hr time points. High albumin, phosphorus, and creatinine phosphokinase were noted preprocedure and persisted throughout the duration of the study. All other indicators remained within normal ranges after PRVI.

\section{Ex Vivo Quantitative Analysis (Figs. 4 and 5)}

SNRs of lipiodol deposition was increased in the pancreatic body/tail in comparison with the pancreatic head

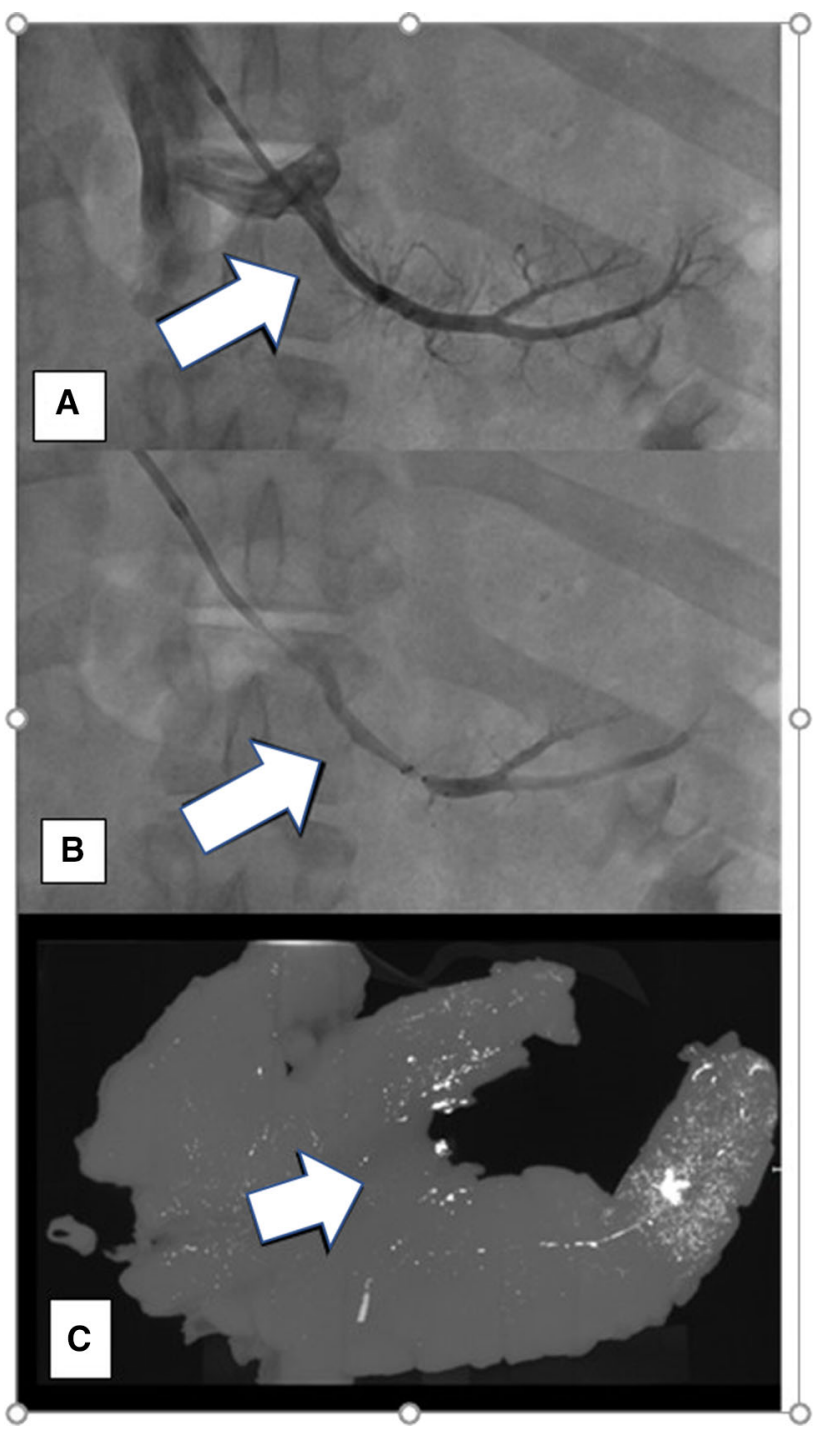

Fig. 2 Transhepatic venous delivery into the pancreatic veins draining the body/tail (White arrow = body). A Contrast venography (anteroposterior view) of the pancreatic vein draining the pancreatic body (white arrow). Pancreatic vein is arising off the splenic vein. B Placement of microvalvular system (white arrow) into the pancreatic vein to isolate the pancreatic body. C Ex vivo threedimensional volumetric coronal MIP (micro-CT) of the pancreas after lipiodol injection. A circular region of interest (ROI) was placed in the area of maximum density (yellow circle) and in background tissue (white arrow) to calculate SNR

$(p=0.009)$. The SNR of the pancreatic tissue was shown to be significantly higher in the pancreas versus extra pancreatic tissue (gallbladder and duodenum, $p=0.0001$ ).

\section{Histopathological Analysis (Fig. 6)}

Pancreatic tissue architecture was intact without any significant inflammation, ischemia, edema, or tissue damage. 


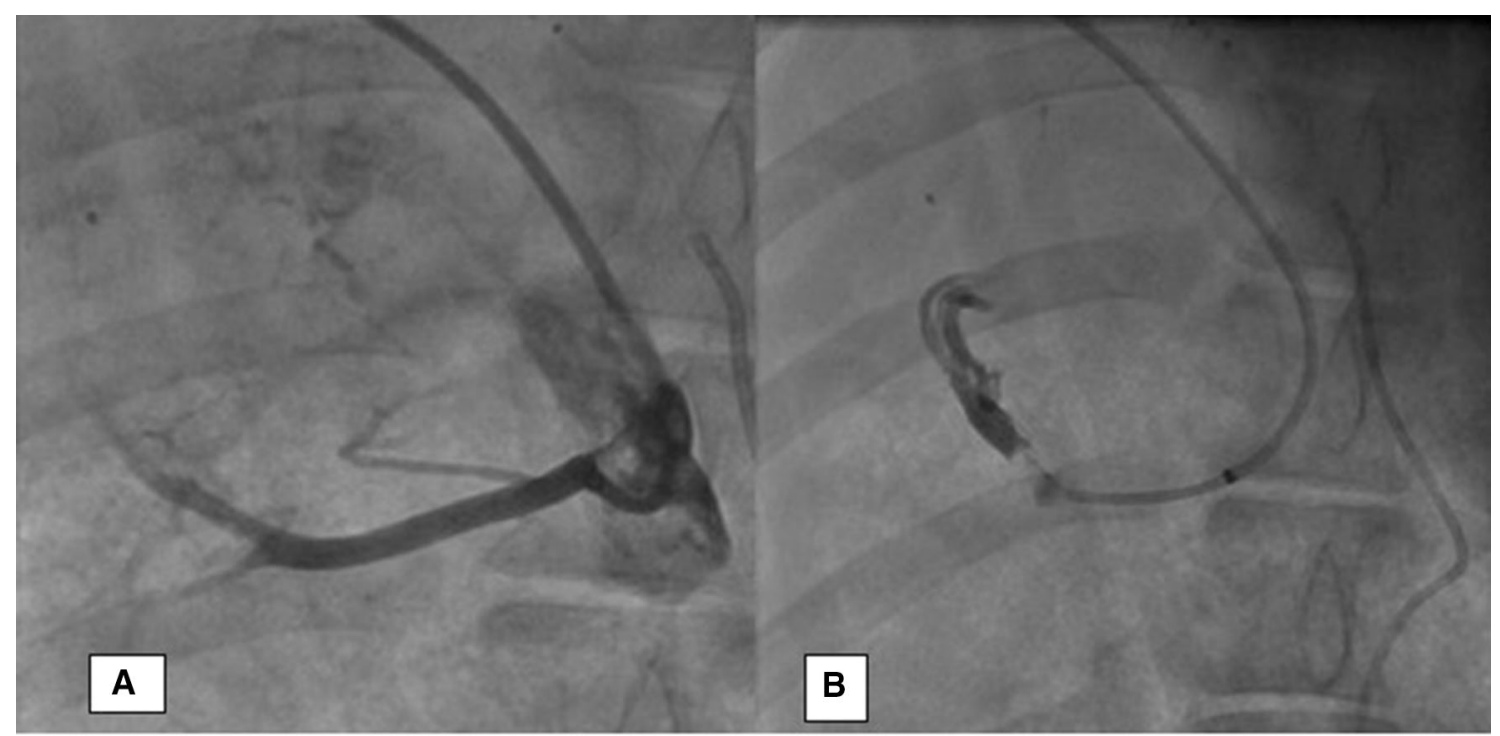

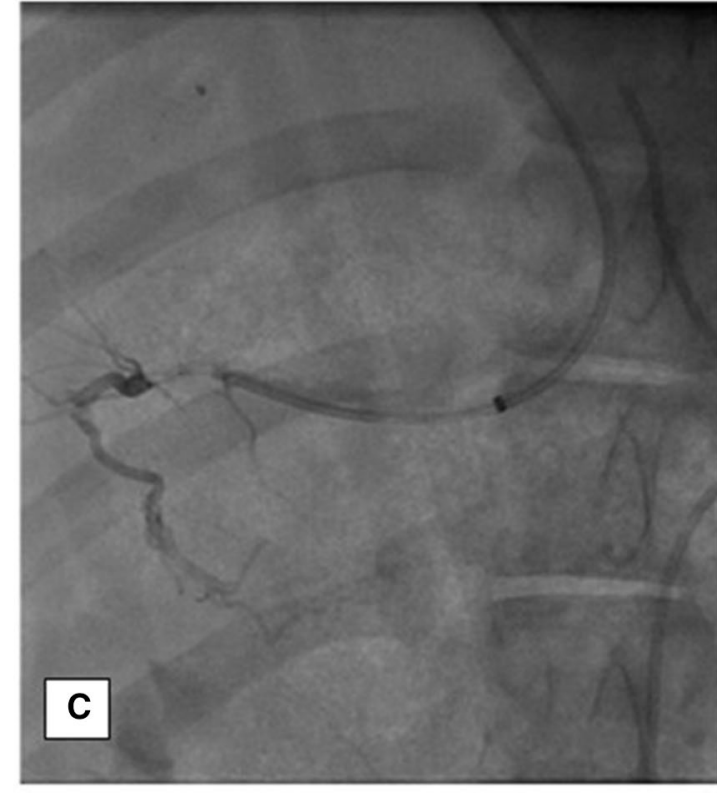

Fig. 3 Transhepatic venous delivery into the pancreatic veins draining the head. A Contrast venography (anteroposterior view) of the superior pancreato-duodenal branch off the portal vein. B Cannulation of duodenal branch with microvalvular system prior to

\section{Discussion}

The main finding of our study is that the PEDD approach in the pancreatic venous system is technically feasible in a large animal model. Using a combination of radiopaque lipiodol, a microvalvular infusion system, and 3D volumetric imaging with micro-CT, we confirmed that PRVI of agents into the pancreatic tissues is achievable. In addition, based on SNR calculations, there was a statistically significant increased amount of lipiodol in the targeted pancreatic body versus the pancreatic head and similarly versus extra pancreatic tissue. Thus, PEDD may serve as a

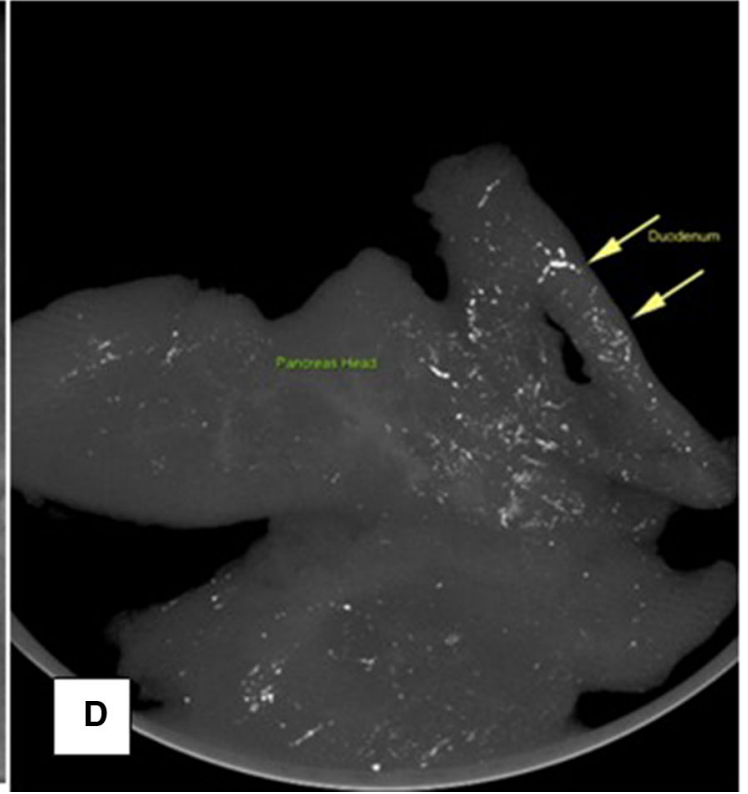

embolization with gelfoam. C Cannulation of pancreatic head vein with microvalvular system. D Ex vivo three-dimensional volumetric coronal MIP (micro-CT) of pancreatic head and duodenum

viable alternative for locoregional delivery of therapeutics into pancreatic tissue.

All prior vascular efforts targeting pancreatic cancer have utilized an arterial approach via the celiac and/or splenic arteries [14, 15]. As the feeding arteries directly into the tumor are often too small to catheterize, the current IA strategy is to flood and saturate the arterial system closest to the tumor bed [16]. The IA methodology requires extensive catheterization and embolization of multiple vessels in order to "skeletonize" the arterial system so the therapeutic agent only travels in one of the main arteries (celiac, gastroduodenal or splenic artery) adjacent to the 
Table 1 Laboratory Values Pre- and Post-Treatment at 5 Days

\begin{tabular}{|c|c|c|c|c|c|c|c|c|c|c|}
\hline \multirow[t]{2}{*}{ Parameter } & \multirow[t]{2}{*}{ Reference } & \multirow[t]{2}{*}{ Unit } & \multicolumn{4}{|l|}{ Animal 1} & \multicolumn{4}{|l|}{ Animal 2} \\
\hline & & & Pre-procedure & Post-procedure & $24 \mathrm{~h}$ & $96 \mathrm{~h}$ & Pre-procedure & Post-procedure & $24 \mathrm{~h}$ & $96 \mathrm{~h}$ \\
\hline Total protein & $5.0-7.0$ & $\mathrm{~g} / \mathrm{dL}$ & 5.8 & 5.1 & 5.5 & 5.5 & 6.2 & 6.2 & 6.5 & 6.2 \\
\hline Albumin & $2.4-3.3$ & $\mathrm{~g} / \mathrm{dL}$ & 3.7 & 3.2 & 3.6 & 3.4 & 3.6 & 3.5 & 3.9 & 3.6 \\
\hline AST (SGOT) & $10-100$ & $\mathrm{IU} / \mathrm{L}$ & 22 & 22 & 55 & 90 & 24 & 26 & 34 & 28 \\
\hline ALT (SGPT) & $10-100$ & $\mathrm{IU} / \mathrm{L}$ & 31 & 26 & 30 & 46 & 26 & 25 & 27 & 36 \\
\hline Alk phosphatase & $100-250$ & $\mathrm{IU} / \mathrm{L}$ & 233 & 231 & 225 & 187 & 115 & 116 & 122 & 115 \\
\hline GGT & & $\mathrm{IU} / \mathrm{L}$ & 38 & 43 & 41 & 39 & 30 & 28 & 32 & 37 \\
\hline Total bilirubin & $0.0-1.0$ & $\mathrm{mg} / \mathrm{dL}$ & 0.3 & 0.5 & 0.1 & 0.1 & 0.2 & 0.3 & 0.1 & 0.1 \\
\hline Phosphorus & $3.0-5.5$ & $\mathrm{mg} / \mathrm{dL}$ & 9.1 & 9.6 & 7.2 & 8.1 & 8.1 & 7.6 & 6.9 & 8.1 \\
\hline Glucose & $70-120$ & $\mathrm{mg} / \mathrm{dL}$ & 85 & 70 & 102 & 91 & 83 & 86 & 95 & 94 \\
\hline Calcium & $7.2-11.5$ & $\mathrm{mg} / \mathrm{dL}$ & 10.3 & 9.7 & 10 & 10.2 & 10.2 & 10.5 & 10 & 10.4 \\
\hline Magnesium & & $\mathrm{mEq} / \mathrm{L}$ & 1.5 & 1.5 & 1.7 & 1.7 & 1.4 & 1.2 & 1.8 & 1.7 \\
\hline Amylase & $1000-2500$ & $\mathrm{IU} / \mathrm{L}$ & 2,186 & 1,925 & 2,030 & 1,908 & 2,396 & 2,315 & 2,137 & 1,637 \\
\hline CPK & $100-400$ & $\mathrm{IU} / \mathrm{L}$ & 3,988 & 3,541 & 6,123 & 7,108 & 1,033 & 954 & 1,612 & 541 \\
\hline LDH & & $\mathrm{IU} / \mathrm{L}$ & 426 & 371 & 601 & 1,137 & 367 & 342 & 457 & 439 \\
\hline WBC & $9.6-25.2$ & $10^{\wedge} 3 / \mathrm{uL}$ & 15.4 & 15.1 & 13.9 & 16 & 13.2 & 14.2 & 12.1 & 12.4 \\
\hline $\mathrm{RBC}$ & $4.9-7.9$ & $10^{\wedge} 6 / \mathrm{uL}$ & 6.4 & 6 & 6.7 & 5.9 & 6.6 & 6.5 & 6.9 & 6.5 \\
\hline HGB & $8.1-11.9$ & $\mathrm{~g} / \mathrm{dL}$ & 11.6 & 10.9 & 12.2 & 10.5 & 11.2 & 11 & 11.8 & 10.9 \\
\hline HCT & $28-40$ & $\%$ & 36 & 34 & 40 & 35 & 35 & 35 & 39 & 37 \\
\hline Platelet count & $200-800$ & $10^{\wedge} 3 / \mathrm{uL}$ & 214 & 251 & 229 & 137 & 266 & 233 & 449 & 376 \\
\hline
\end{tabular}

$A L T$ alanine aminotransferase, GGT gamma glutamyltransferase, AST aspartate aminotransferase, INR international normalized ratio, $C P K$ creatinine phosphokinase, $W B C$ white blood count, $R B C$ red blood cell count, $H G B$ Hemoglobin $\mathrm{HCT}=$ hematocrit.

tumor $[5,6,16]$. Furthermore, as the systemic exposure is significant, these patients often undergo multiple repeated procedures with lower doses in order to achieve a sufficient therapeutic index [17].

Nevertheless, clinical trials have shown that regional IA infusion with gemcitabine may have the potential to improve the response and resectability rates for advanced pancreatic cancer in highly selected patients. A metaanalysis of 298 patients (both stage 3 and 4) undergoing IA delivery using gemcitabine showed a survival benefit of $30 \%$ at 1 year when compared with systemic gemcitabine [6]. In addition, Rosemurgy and colleagues reported a study in which 20 subjects with locally advanced PDAC received 100 catheter-directed IA infusions, with the number of procedures reflective of the technical challenges. In 15 evaluable patients, the 1-year survival rate was $60 \%$ and $58 \%$ of the population had CA19-9 reductions [5]. However, all these studies are significantly hampered by serious adverse events which include sepsis, arterial dissection, hyperglycemia, and neutropenia which are usually attributable to non-target delivery [5]. In addition, the studies did not contain control arms and likely reflect a high degree of selection bias, while the follow-up times were too short to draw meaningful outcome conclusions.
Arterial infusion methods are fundamentally limited because pancreatic tumors have poor arterial perfusion compared to surrounding healthy tissue due to the desmoplastic stroma $[18,19]$. Thus, flow directed therapies such as IA infusions often lead to non-target delivery. Despite the use of extensive embolization techniques to isolate the vasculature, the inability to target the tumors remains significant [7, 16]. Furthermore, hypoxia and decreased vascularity in combination with elevated interstitial pressures prevent chemotherapeutic agents from entering the tumor $[15,20]$.

Our pilot results suggest the alternative transvenous route of delivering chemotherapy to PDAC, coupled with PEDD, may overcome some of the limitations associated with the IA approach. In comparison with systemic intravenous and locoregional IA therapies, PEDD in the venous system does not rely on the limited arterial supply and instead takes advantage of the more easily accessible venous branches which drain the pancreatic tumor [13].

Although this is a unique approach for the pancreas, this methodology has been utilized in other organ systems where there is limited arterial access. For example, in the setting of cellular/gene therapies, retrograde transvenous methodology has been described in the targeting of myocardium and extremity musculature associated with 


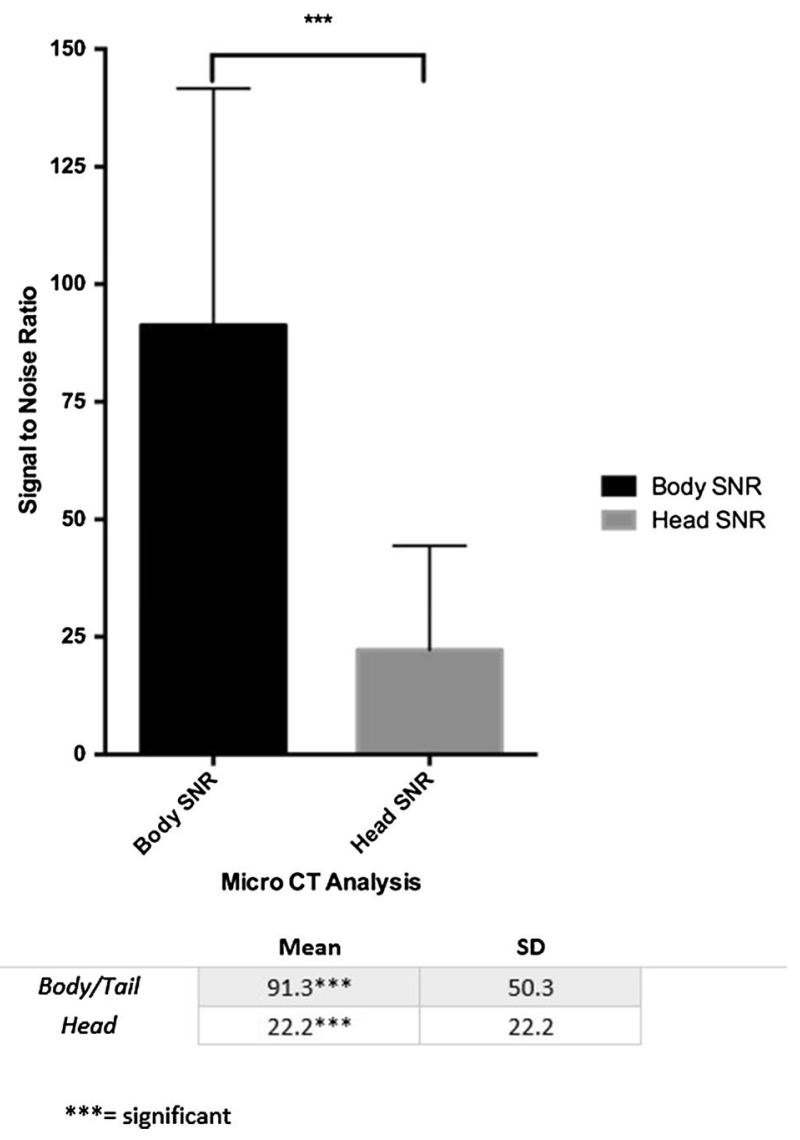

Fig. 4 Quantitative assessment of lipiodol distribution utilizing SNR in the pancreatic body vs head based on micro-CT analysis

coronary artery disease and muscular dystrophy, respectively [21-23]. In this technique, therapeutics are delivered in a retrograde fashion directly into the myocardium or target extremity via the coronary sinus or the main venous drainage of the affected extremity. Thus, there is a growing awareness on utilizing this approach for locoregional therapies.

Utilizing 3D volumetric micro-CT imaging analysis (isotropic resolution of 70um) and the mathematical calculation of SNR, we were able to accurately depict and quantify deposition of lipiodol in various parts of the pancreas. In our study, the SNR in the pancreatic body was 91.3 (+ /50.3) compared to the non-target pancreatic head, which was $22.2( \pm 22.2)$. Although SNR calculations with lipiodol are a crude surrogate for tissue-level concentrations, it appears that highly concentrated and targeted delivery is achievable. Although we did notice delivery of lipiodol in extra pancreatic tissue such as duodenum and gallbladder during pancreatic head infusions, the delivery to the pancreatic tissue was still significantly higher $(p=0.0001)$. Of note, we did not notice any lipiodol within the gastric mucosa. Lastly, the imaging data and biochemical analysis at 4 days suggests the targeting via

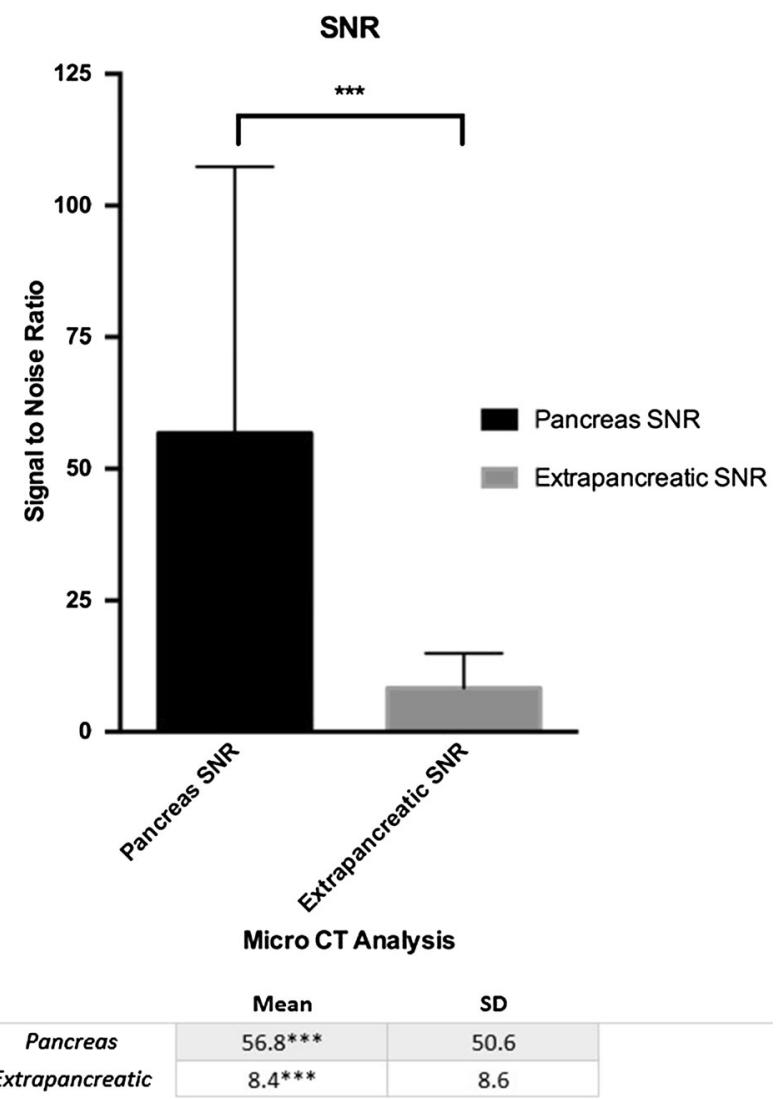

Fig. 5 Quantitative assessment of lipiodol distribution utilizing SNR in the pancreas vs. extra pancreatic tissue based on micro-CT analysis

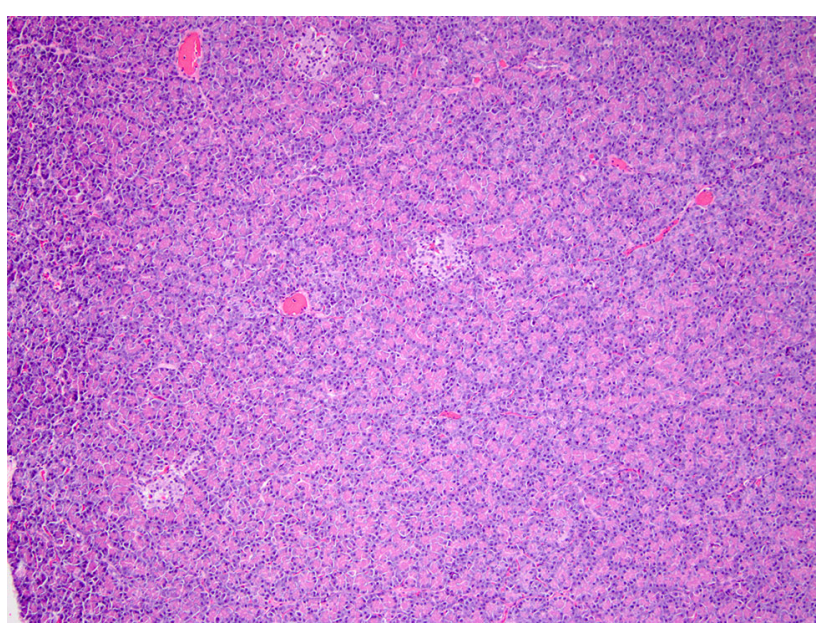

Fig. 6 Hematoxylin and eosin (H\&E) histology of the pancreatic body (infusion site); microscopically evaluated thin sections of the pancreas show no evidence of necrosis, fibrosis, or inflammation

PEDD is both durable in terms of therapy remaining in the target tissue and is a strong indicator that the method successfully deposited therapy into regions of poorly perfused tissue. 


\section{Limitations}

Our study has several limitations. First, only two animals of the 10 underwent a 4-day survival. Therefore, the longterm in vivo effects of this method have only undergone a limited assessment with this investigation. Further analysis would require a longer-term survival with comparison of transvenous delivery to systemic chemotherapeutic regimens serving as controls. In addition, a refined quantitative estimation of the distribution of radiopaque lipiodol in a tumor model was not performed. The opportunity to test this type of technique in an oncology setting would be of interest and provide important data both on infusion and locoregional response to therapy. However, a workable porcine model of pancreatic cancer, termed Oncopig, is undergoing development/validation and may be an option for future studies [24]. Finally, we speculate that there may be some technical challenges that need to be further elucidated in the clinical setting. As shown in our study, this technique may have the potential for non-target delivery, particularly in inoperable neoplasms. As PDAC tumors are known to invade mesenteric vasculature, retrograde infusion could potentially deliver a therapeutic to draining viscera such as small bowel. Thus, in these types of tumors, a more detailed analysis of the involved visceral vessels needs to be well understood prior to the procedure. For the time being, we intend to exclude patients with mesenteric or portal venous occlusion. Finally, should this technique may require multiple treatments, the added risk of repeated portal venous punctures must be weighed in designing therapeutic plans [24].

\section{Conclusion}

In summary, PRVI utilizing the PEDD approach is technically feasible and associated with no significant organ damage in this pilot animal study. The ability to deliver therapy selectively into the pancreatic head and body is possible with improved targeting to the pancreatic body.

\section{Compliance with Ethical Standards}

Conflict of interest Aravind Arepally, James Chomas, and Steven Katz have ownership interests in TriSalus Life Sciences. Aravind Arepally, James Chomas, and David Jaroch have intellectual property interests in TriSalus Life Sciences. David Jaroch is an employee of TriSalus Life Sciences and has ownership interests.

Human and Animal Rights All procedures performed in studies involving human participants were in accordance with the ethical standards of the institutional and/or national research committee and with the 1964 Helsinki Title Page declaration and its later amendments or comparable ethical standards. For this type of study, formal consent is not required.
Open Access This article is licensed under a Creative Commons Attribution 4.0 International License, which permits use, sharing, adaptation, distribution and reproduction in any medium or format, as long as you give appropriate credit to the original author(s) and the source, provide a link to the Creative Commons licence, and indicate if changes were made. The images or other third party material in this article are included in the article's Creative Commons licence, unless indicated otherwise in a credit line to the material. If material is not included in the article's Creative Commons licence and your intended use is not permitted by statutory regulation or exceeds the permitted use, you will need to obtain permission directly from the copyright holder. To view a copy of this licence, visit http://creativecommons. org/licenses/by/4.0/.

\section{References}

1. Siegel RL, Miller KD, Jemal A. Cancer Statistics, 2017. CA Cancer J Clin. 2017;67(1):7-30.

2. Von Hoff DD, Ervin T, Arena FP, Chiorean EG, Infante J, Moore $\mathrm{M}$, et al. Increased survival in pancreatic cancer with nab-paclitaxel plus gemcitabine. N Engl J Med. 2013;369(18):1691-703.

3. Hackert T, Sachsenmaier M, Hinz U, Schneider L, Michalski $\mathrm{CW}$, Springfeld C, et al. Locally advanced pancreatic cancer: neoadjuvant therapy with folfirinox results in resectability in $60 \%$ of the patients. Ann Surg. 2016;264(3):457-63.

4. Mokdad AA, Minter RM, Zhu H, Augustine MM, Porembka MR, Wang SC, et al. Neoadjuvant therapy followed by resection versus upfront resection for resectable pancreatic cancer: a propensity score matched analysis. J Clin Oncol. 2017;35(5):515-22.

5. Rosemurgy AS, Ross SB, Vitulli PL, Malek R, Li J, Agah R. Safety study of targeted and localized intra-arterial delivery of gemcitabine in patients with locally advanced pancreatic adenocarcinoma. J Pancreat Cancer. 2017;3(1):58-655.

6. Liu F, Tang Y, Sun J, Yuan Z, Li S, Sheng J, et al. Regional intraarterial vs. systemic chemotherapy for advanced pancreatic cancer: a systematic review and meta-analysis of randomized controlled trials. PLoS ONE. 2012;7(7):e40847.

7. Liu X, Yang X, Zhou G, Chen Y, Li C, Wang X. Gemcitabinebased regional intra-arterial infusion chemotherapy in patients with advanced pancreatic adenocarcinoma. Medicine (Baltimore). 2016;95(11):e3098.

8. Uzunparmak B, Sahin IH. Pancreatic cancer microenvironment: a current dilemma. Clin Transl Med. 2019;8(1):2.

9. Koay EJ, Truty MJ, Cristini V, Thomas RM, Chen R, Chatterjee $\mathrm{D}$, et al. Transport properties of pancreatic cancer describe gemcitabine delivery and response. $\mathrm{J}$ Clin Invest. 2014;124(4):1525-36.

10. Longo V, Brunetti O, Gnoni A, Cascinu S, Gasparini G, Lorusso $\mathrm{V}$, et al. Angiogenesis in pancreatic ductal adenocarcinoma: A controversial issue. Oncotarget. 2016;7(36):58649-58.

11. Provenzano PP, Hingorani SR. Hyaluronan, fluid pressure, and stromal resistance in pancreas cancer. $\mathrm{Br} \mathrm{J}$ Cancer. 2013;108(1):1-8.

12. Stylianopoulos T, Martin JD, Snuderl M, Mpekris F, Jain SR, Jain RK. Coevolution of solid stress and interstitial fluid pressure in tumors during progression: implications for vascular collapse. Cancer Res. 2013;73(13):3833-41.

13. Javadi S, Karbasian N, Bhosale P, de Castro FS, Le O, Katz MH, et al. Imaging findings of recurrent pancreatic cancer following resection. Abdom Radiol (NY). 2018;43(2):489-96.

14. Piscaglia F, Ogasawara S. Patient selection for transarterial chemoembolization in hepatocellular carcinoma: importance of benefit/risk assessment. Liver Cancer. 2018;7(1):104-19. 
15. Schorn S, Demir IE, Reyes CM, Saricaoglu C, Samm N, Schirren $\mathrm{R}$, et al. The impact of neoadjuvant therapy on the histopathological features of pancreatic ductal adenocarcinoma - A systematic review and meta-analysis. Cancer Treat Rev. 2017;55:96-106

16. Homma H, Doi T, Mezawa S, Takada K, Kukitsu T, Oku T, et al. A novel arterial infusion chemotherapy for the treatment of patients with advanced pancreatic carcinoma after vascular supply distribution via superselective embolization. Cancer. 2000;89(2):303-13.

17. Hong GB, Zhou JX, Sun HB, Li CY, Song LQ. Continuous transarterial infusion chemotherapy with gemcitabine and 5-Fluorouracil for advanced pancreatic carcinoma. Asian Pac J Cancer Prev. 2012;13(6):2669-733.

18. Nguyen NC, Taalab K, Osman MM. Decreased blood flow with increased metabolic activity: a novel sign of pancreatic tumor aggressiveness. Clin Cancer Res. 2010;16(1):367 author reply 567.

19. Michalski CW, Erkan M, Friess H, Kleeff J. Tumor metabolism to blood flow ratio in pancreatic cancer: helpful in patient stratification? Future Oncol. 2010;6(1):13-5.

20. Heinrich S, Lang H. Neoadjuvant Therapy of Pancreatic Cancer: Definitions and Benefits. Int J Mol Sci. 2017;18(8):1-16.
21. George JC, Goldberg J, Joseph M, Abdulhameed N, Crist J, Das $\mathrm{H}$, et al. Transvenous intramyocardial cellular delivery increases retention in comparison to intracoronary delivery in a porcine model of acute myocardial infarction. J Interv Cardiol. 2008;21(5):424-31.

22. Fan Z, Kocis K, Valley R, Howard JF, Chopra M, An H, et al. Safety and feasibility of high-pressure transvenous limb perfusion with $0.9 \%$ saline in human muscular dystrophy. Mol Ther. 2012;20(2):456-61.

23. Fan Z, Kocis K, Valley R, Howard JF Jr, Chopra M, Chen Y, et al. High-pressure transvenous perfusion of the upper extremity in human muscular dystrophy: a safety study with $0.9 \%$ saline. Hum Gene Ther. 2015;26(9):614-21.

24. Schachtschneider KM, Schwind RM, Newson J, Kinachtchouk N, Rizko M, Mendoza-Elias N, et al. The oncopig cancer model: an innovative large animal translational oncology platform. Front Oncol. 2017;7:190.

Publisher's Note Springer Nature remains neutral with regard to jurisdictional claims in published maps and institutional affiliations. 